\title{
Multiplexed immunoassay-based serum cytokine profiling for potential prognosis predictors in patients with metastatic breast cancer
}

\author{
Hongyan Huang, Yanbin Zhang, Sha Li, Jing Zhao, Xiaoli Wang, Xinna Zhou, Jun Ren \\ Department of Oncology, Beijing Shijitan Hospital of Capital Medical University, Beijing 100038, China \\ Contributions: (I) Conception and design: H Huang; (II) Administrative support: J Ren, X Zhou, X Wang; (III) Provision of study materials or \\ patients: H Huang, J Ren; (IV) Collection and assembly of data: S Li, J Zhao; (V) Data analysis and interpretation: Y Zhang, H Huang; (VI) \\ Manuscript writing: All authors; (VII) Final approval of manuscript: All authors. \\ Correspondence to: Hongyan Huang, MD, PhD. Department of Oncology, Beijing Shijitan Hospital of Capital Medical University, 10 Tieyi Road, \\ Beijing 100038, China. Email: hhongy1999@126.com.
}

\begin{abstract}
Background: Although the patients suffering from metastatic breast cancer (MBC) have experienced considerably wide survival ranges, reliable biomarkers for its prognosis have remained unidentified. This study attempted to profile the serum cytokine candidates that could potentially be used to predict the prognosis of MBC patients.

Methods: By using the ProcartaPlex Multiplex Immunoassay, a total of 45 cytokines were assayed in sera from 18 MBC patients before any treatment.

Results: Thirty-three types of cytokines were cross-detectable in more than $50 \%$ of the patients and thus were acceptable for a further analysis. Univariate analysis uncovered 10 sera cytokines significantly associated with MBC patient death. Among them, interleukin 8 (IL-8), fibroblast growth factor (FGF2), hepatocyte growth factor (HGF) and VEGF-A were reported, and 6 cytokines which included NGF- $\beta$, IFN- $\gamma$, IP-10, IL-18, MCP-1 and MIP-1 $\beta$ were identified for the first time as MBC death-correlated serum factors.

Conclusions: Identification of these cytokines would facilitate developing novel serum biomarkers for $\mathrm{MBC}$, favoring multiplexed serum cytokine profiling a promising method in predicting the outcome of MBC patients.
\end{abstract}

Keywords: Cytokine profiling; prognosis; metastatic breast cancer (MBC)

Submitted May 06, 2018. Accepted for publication Nov 21, 2018.

doi: $10.21037 /$ tcr.2018.11.29

View this article at: http://dx.doi.org/10.21037/tcr.2018.11.29

\section{Introduction}

Breast cancer represents the most common malignancy for women. The majority of breast cancer patients diagnosed at an early disease stage have a long-term survival time after standard therapy which includes surgery and adjuvant therapy. However, the patients with distant metastasis at their initial diagnosis, and those that develop metastatic disease during or after standard therapy are generally incurable. In these cases, the primary treatment goal is symptom palliation. Although it is widely accepted that metastatic breast cancer (MBC) occurs in 20-30\% of women with breast cancer with a median survival ranging from 2 to 3 years (1-3), MBC patients usually experience a very wide range of survival time, ranging from less than one year to more than ten years, which indicates that the prognosis of MBC is significantly diverse (4). The diversity in the outcomes for MBC patients highlights the need to identify the markers that contribute to improved prognosis. Serum biomarkers are preferred by clinics because they are capable of being dynamically monitored in a noninvasive way.

Cytokines are polypeptide growth factors produced 
by most nucleated cells in the body (5). They work as the major mediator for the interactions between tumor cells and their microenvironments, which plays an important role in the development and progression of solid tumors, including breast cancer (6). To some extent, the cytokine levels in the serum can read out their expressions in the tumors; therefore, they are regarded as valuable prognostic biomarkers for cancer patients. For example, high serum IL-37 level was associated with unfavorable prognosis of epithelial ovarian cancer patients (7), and the serum levels of interleukin 8 (IL-8), macrophage inflammatory protein (MIP)-1 $\alpha$, MIP-1 $\beta$ were significantly higher in breast cancer patients (8). Nevertheless, despite sporadic reports on individual factors, a comprehensive profiling of multiple serum cytokines has not as of yet been reported for MBC patients. ProcartaPlex Multiplex Immunoassays, which incorporate magnetic microsphere technology to enable the simultaneous detection and quantitation of up to 100 protein targets in diverse matrices, allow us to systemically identify potential novel biomarkers in a single sample of serum for MBC patients.

By taking advantage of the ProcartaPlex technique, we performed a retrospective study in which 45 cytokines were assayed in 18 patients with MBC. Our work identified a group of cytokines in sera that may potentially serve as novel prognostic biomarkers for MBC patients, and favored serum cytokine profiling as a promising method in assisting the prediction of $\mathrm{MBC}$ prognosis.

\section{Methods}

\section{Study design and patients}

We performed a retrospective analysis of women with histologically confirmed MBC over a 1-year period in our clinic in China. The study was approved by the Institutional Ethical Committee of the Beijing Shijitan Hospital. All participants provided written informed consent for the research use of blood and the outcome data. Blood samples were collected before treatment and processed following standard procedures and cryopreserved without thawing before this analysis.

\section{Serum cytokine detection}

The concentrations of cytokines in the serum were determined using the ProcartaPlex Human Cytokine 45plex immunoassay kits (EPX450-12171-901, eBioscience) following the manufacturer's recommendations. The 45 cytokines and growth factors assayed are brainderived neurotrophic factor (BDNF), epidermal growth factor (EGF), eotaxin, fibroblast growth factor (FGF2), granulocyte-macrophage colony stimulating factor (GMCSF), growth-related oncogene protein- $\alpha$ (GRO- $\alpha$ ), hepatocyte growth factor (HGF), IFN- $\alpha$, IFN- $\gamma$, IL-1RA, IL-1 $\alpha$, IL-1 $\beta$, IL-2, IL-4, IL-5, IL-6, IL-7, IL-8, IL-9, IL10, IL-12p70, IL-13, IL-15, IL-17A, IL-18, IL-21, IL22, IL-23, IL-27, IL-31, IFN- $\gamma$-inducible protein 10 (IP10, CXCL10), leukemia inhibitory factor (LIF), monocyte chemotactic protein 1 (MCP-1), macrophage inflammatory protein type $1 \beta$ (MIP-1 $\beta$, CCL4), MIP-1 $\alpha$, NGF- $\beta$, chemokine (C-C motif) ligand 5 (CCL5/RANTES), platelet-derived growth factor-BB (PDGF-BB), placenta growth factor-1 (PIGF-1), stem cell factor (SCF), stromal cell-derived factor 1 (SDF-1 $\alpha)$, tumor necrosis factor- $\alpha$ $(\mathrm{TNF}-\alpha), \mathrm{TNF}-\beta$, vascular endothelial growth factor-A (VEGF-A), and VEGF-D.

\section{Statistical analysis}

Statistical analysis was performed using SPSS 20.0 software (SPSS, Chicago, IL, USA). The overall survival (OS) was defined as time from when blood sample was first collected after admission to the clinic, until death due to any cause or the date of last contact with the patient or any family member. The results were analyzed using the MannWhitney $U$ test. The prognostic value of the cytokines was studied using univariate analysis. $\mathrm{P}$ value $<0.05$ was considered statistically significant, and all values were onesided.

\section{Results}

\section{Patient characteristics}

From April 2015 to April 2016, 18 patients with histologically confirmed MBC were enrolled in this retrospective study. The baseline characteristics of the 18 patients are summarized in Table 1. The median age was 47.4 years (range, 27.6-90.1 years). Most patients were hormone receptor-negative (55.6\%) and HER2-positive (55.6\%). The most common sites of metastasis were bone $(72.2 \%)$, soft tissue $(61.1 \%)$ and the lung $(55.6 \%)$. The majority of patients had received prior anthracyclinebased chemotherapy (84.4\%), with more than half $(54.7 \%)$ receiving both prior anthracyclines and taxanes. 
Table 1 Patient characteristics

\begin{tabular}{|c|c|}
\hline Characteristic & Number \\
\hline Median age, years (range) & $47.4(27.6-90.1)$ \\
\hline \multicolumn{2}{|l|}{ Menopausal status, n (\%) } \\
\hline Pre & $11(61.1)$ \\
\hline Post & 7 (38.9) \\
\hline \multicolumn{2}{|l|}{ ER/PR status, n (\%) } \\
\hline Positive & $8(44.4)$ \\
\hline Negative & $10(55.6)$ \\
\hline \multicolumn{2}{|l|}{ HER2 status, n (\%) } \\
\hline Positive & $10(55.6)$ \\
\hline Negative & $8(44.4)$ \\
\hline \multicolumn{2}{|l|}{ Metastatic sites, n (\%) } \\
\hline Liver & $3(16.7)$ \\
\hline Lung & $10(55.6)$ \\
\hline Brain & $1(5.6)$ \\
\hline Bone & $13(72.2)$ \\
\hline Soft tissue & $11(61.1)$ \\
\hline Other & $5(27.8)$ \\
\hline \multicolumn{2}{|l|}{ Number of tumor sites, n (\%) } \\
\hline 1 & $1(5.6)$ \\
\hline 2 & $2(11.1)$ \\
\hline$\geq 3$ & $15(83.3)$ \\
\hline \multicolumn{2}{|c|}{ Setting of study treatment, $\mathrm{n}(\%)$} \\
\hline First line & $6(33.3)$ \\
\hline Second line & $6(33.3)$ \\
\hline Greater than second line & $6(33.3)$ \\
\hline
\end{tabular}

ER, estrogen receptor; PR, progesterone receptor.

\section{Serum cytokine levels in patients}

Among the 45 cytokines assayed, 33 cytokines were detectable in more than $50 \%$ of the patients and were acceptable for further analysis. Twelve cytokines which failed to be detected in more than $50 \%$ of the patients were as follow: IL-4, IL-5, GM-CSF, IL-21, IL-22, IL-23, IL-9, INF- $\alpha$, IL- $1 \alpha$, IL-7, TNF- $\beta$, VEGF-D.

\section{Association between cytokine level and prognosis}

All the patients were followed-up for at least one year. Ten
Table 2 Univariate analyses of risk factors for MBC patient death

\begin{tabular}{|c|c|c|c|c|}
\hline Variable & Status & $\mathrm{N}$ & Mean rank & $P$ value \\
\hline \multirow[t]{2}{*}{ IL-18 } & Live & 8 & 5.5 & 0.003 \\
\hline & Dead & 10 & 12.7 & \\
\hline \multirow[t]{2}{*}{$N G F-\beta$} & Live & 8 & 6.5 & 0.032 \\
\hline & Dead & 10 & 11.9 & \\
\hline \multirow[t]{2}{*}{ HGF } & Live & 8 & 6 & 0.012 \\
\hline & Dead & 10 & 12.3 & \\
\hline \multirow[t]{2}{*}{ IP-10 } & Live & 8 & 6.38 & 0.027 \\
\hline & Dead & 10 & 12 & \\
\hline \multirow[t]{2}{*}{ MCP-1 } & Live & 8 & 6.38 & 0.027 \\
\hline & Dead & 10 & 12 & \\
\hline \multirow[t]{2}{*}{ IFN- $\gamma$} & Live & 8 & 6.06 & 0.012 \\
\hline & Dead & 10 & 12.25 & \\
\hline \multirow[t]{2}{*}{ FGF2 } & Live & 8 & 6.13 & 0.016 \\
\hline & Dead & 10 & 12.2 & \\
\hline \multirow[t]{2}{*}{ VEGF-A } & Live & 8 & 5.38 & 0.002 \\
\hline & Dead & 10 & 12.8 & \\
\hline \multirow[t]{2}{*}{ IL-8 } & Live & 8 & 5.25 & 0.001 \\
\hline & Dead & 10 & 12.9 & \\
\hline \multirow[t]{2}{*}{ MIP-1 $\beta$} & Live & 8 & 5.88 & 0.009 \\
\hline & Dead & 10 & 12.4 & \\
\hline
\end{tabular}

MBC, metastatic breast cancer.

of them died while 8 of them survived as of the last followup. The patients were divided into 2 groups according to their survival status. The association of cytokines level with survival status in all 18 patients was analyzed. As a result, from univariate analysis, MBC patient death was found to be significantly correlated with a group of cytokines including INF- $\gamma$, IL-18, NGF- $\beta$, FGF2, HGF, VEGF-A, IL-8, IP-10, MCP-1, MIP-1 $\beta$ (Table 2, Figure 1). Due to the limited sample size, the multivariate analysis was not performed.

\section{Discussion}

There have been great efforts made to identify the prognostic markers for breast cancer, with a majority of the studies focusing on markers discriminating cancer patients from healthy individuals, or metastatic cancer from 

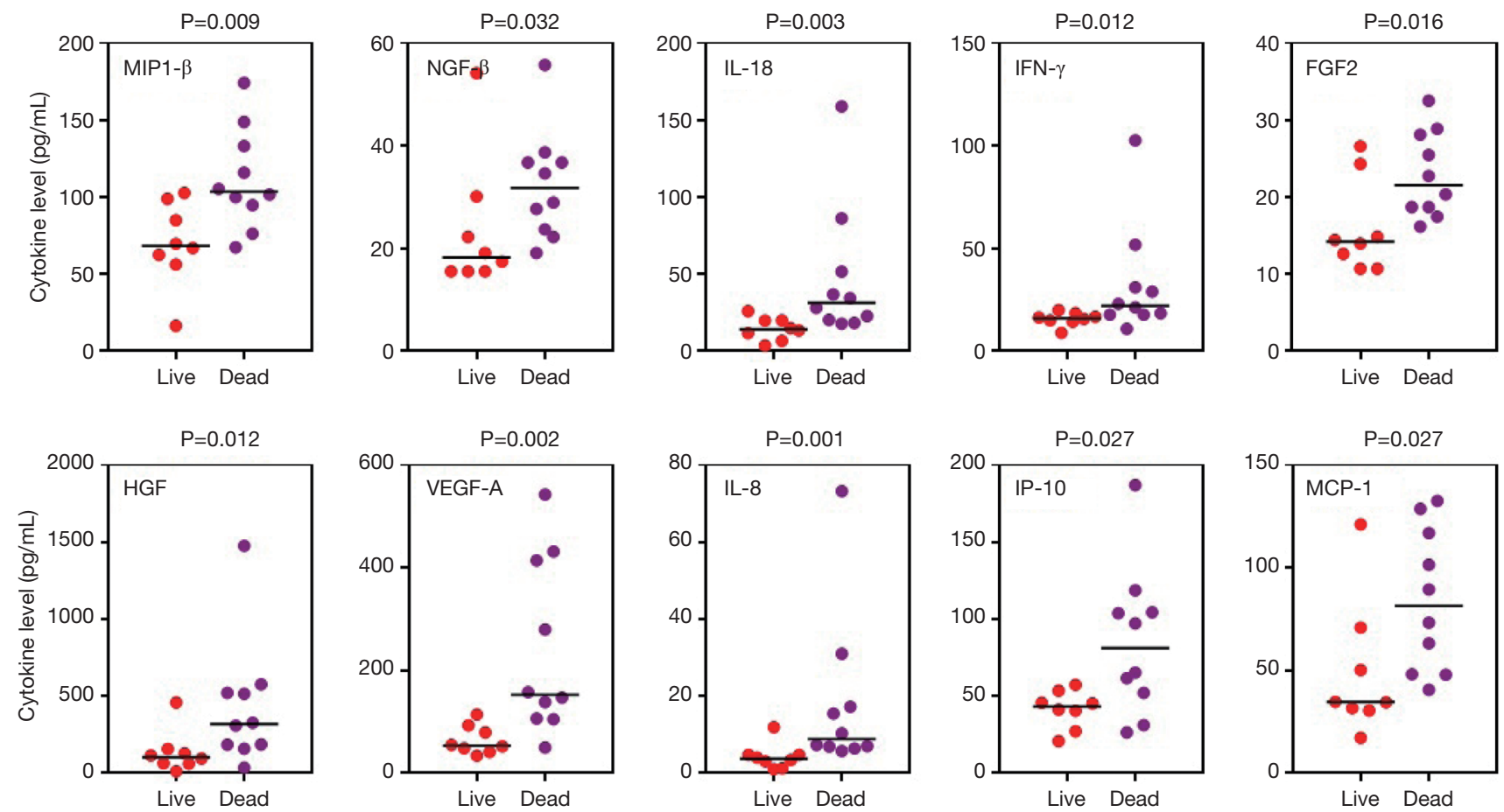

Figure 1 Scatter plot of cytokine levels in patients with metastatic breast cancer. $\mathrm{N}_{\text {live }}=8, \mathrm{~N}_{\text {dead }}=10$; $\mathrm{P}$ values for each comparison are indicated.

localized lesion. Research on identifying the prognostic factors to discriminate long-term survival from short-term death within $\mathrm{MBC}$ patients has been rather limited. In fact, clinical statistics indicated that some of the MBC patients could survive more than 10 years, while some only lived less than 1 year (4). This suggests that these two groups of people are internally different, and may be distinguished from each other at the molecular level.

To this end, we used the ProcartaPlex Immunoassays to simultaneously analyze 45 different cytokines in the serum samples from the MBC patients, who had been classified into 2 groups based on their survival statuses. Our data indicated that 10 cytokines in sera were significantly associated with MBC death. Based on their prior implications in $\mathrm{MBC}$ prognosis, these cytokines were roughly classified into 3 groups:

The $1^{\text {st }}$ group of cytokines includes HGF, IL-8, FGF2 and VEGF, whose levels in serum were previously reported to be associated with MBC prognosis (9-11). For example, serum HGF levels were observed significantly higher in patients with progressive metastasis of breast cancer than those with stable metastasis. The high-HGF patients exhibited much shorter survival rate than those with low HGF levels (11).
The identification of known MBC prognostic cytokines supports the application of our screening technique, even with a limited patient sample size.

The $2^{\text {nd }}$ group of cytokines includes IL-18, MCP-1, MIP-1 $\beta$, IFN- $\gamma$ and IP-10, whose levels in serum were reported to be associated with the prognosis of breast cancers other than MBC (12-15). For example, the levels of serum IL-18 were found to be significantly higher in breast cancer patients than in control subjects, and in metastatic patients than in nonmetastatic patients (12). However, it is unclear whether serum IL-18 was associated with MBC of shorter survival. The similar is true for the other cytokines in this group. The implication of these cytokines in breast cancer is consistent with their roles in predicting MBC outcomes as potentially novel prognostic serum markers reported by this study.

The $3^{\text {rd }}$ group of cytokines consists of only NGF- $\beta$. No association had been reported between its serum level and any breast cancer type, including MBC. Nevertheless, tissue NGF expression was identified as an independent prognostic indicator for relapse-free survival (16). Our result in this study indicated that serum NGF level was associated with the death of MBC patients, which makes it a promising 
candidate of serum prognostic biomarker for MBC.

Though the candidate factors identified in this study are associated with MBC patient survival, the impacts of this finding are considerably compromised by limited patient number. As is well known, the prognosis of MBC patients is influenced by multiple characteristic parameters, including the number of lesions, affected organs, lymph node metastases, statuses of ER, PR, and Her-2 and others. To evaluate the prognostic value of novel factors, a stratification-based multivariate analysis is needed with these known factors included; however, this was not feasible due to the limited number of patients. Therefore, limited patient number might have biasedly led to the generation of false positive candidates in this study. Nevertheless, we did evaluate the association of these prognostic factors with cytokine candidates and patient survival in the current patient setting and failed to reveal significant associations (data not shown). In light of this result, future validation of these cytokine candidates with an expanded patient number is warranted.

To our knowledge, this is the first retrospective study focusing on systemic serum cytokine profiling in Chinese MBC patients. The results indicated that the increased level of 10 cytokines was significantly associated with MBC death, although the independent prognostic value of these cytokines warrants further validation with more MBC patients. Among the cytokines identified, the serum levels of 6 cytokines were reported for the first time to be associated with $\mathrm{MBC}$ death, and constitute novel candidates of prognostic biomarker for MBC patients. Compared with previous studies on a single cytokine, simultaneous measurement of multiple cytokines would considerably improve the diagnostic value; therefore, it would be prudent if future studies investigated the prognostic value of a combined set of the candidate cytokines in patient sera in a larger sample size by multiplexed cytokine profiling.

\section{Conclusions}

Identification of these cytokines would facilitate the development of novel serum biomarkers for MBC, and favor multiplexed serum cytokines profiling as a promising method in predicting the outcome of MBC patients.

\section{Acknowledgments}

Funding: This work was funded by a grant from the Beijing Health System High-level Health Technical Personnel
Training Project (grant number 2015-3-058), the National Natural Science Foundation of China (grant number 81572799), and the Beijing Natural Science Foundation (grant number 7162091).

\section{Footnote}

Conflicts of Interest: All authors have completed the ICMJE uniform disclosure form (available at http://dx.doi. org/10.21037/tcr.2018.11.29). The authors have no conflicts of interest to declare.

Ethical Statement: The authors are accountable for all aspects of the work in ensuring that questions related to the accuracy or integrity of any part of the work are appropriately investigated and resolved. The study was conducted in accordance with the Declaration of Helsinki (as revised in 2013). This study protocol for the collection of samples and clinical information was approved by the Institutional Ethics Committee of Beijing Shijitan Hospital of Capital Medical University (No. 201845). All participants provided written informed consent for the research use of blood and outcome data.

Open Access Statement: This is an Open Access article distributed in accordance with the Creative Commons Attribution-NonCommercial-NoDerivs 4.0 International License (CC BY-NC-ND 4.0), which permits the noncommercial replication and distribution of the article with the strict proviso that no changes or edits are made and the original work is properly cited (including links to both the formal publication through the relevant DOI and the license). See: https://creativecommons.org/licenses/by-nc-nd/4.0/.

\section{References}

1. Flaum LE, Gradishar WJ. Advances in Endocrine Therapy for Postmenopausal Metastatic Breast Cancer. Cancer Treat Res 2018;173:141-54.

2. Anampa J, Sparano JA. New agents for the management of resistant metastatic breast cancer. Expert Opin Pharmacother 2017;18:1815-31.

3. Fernandez Y, Cueva J, Palomo AG, et al. Novel therapeutic approaches to the treatment of metastatic breast cancer. Cancer Treat Rev 2010;36:33-42.

4. Yamamura J, Kamigaki S, Fujita J, et al. The Difference in Prognostic Outcomes Between De Novo Stage IV and Recurrent Metastatic Patients with Hormone 
Receptor-positive, HER2-negative Breast Cancer. In Vivo 2018;32:353-8.

5. Feliciani C, Gupta AK, Sauder DN. Keratinocytes and cytokine/growth factors. Crit Rev Oral Biol Med 1996; 7:300-18.

6. Turley SJ, Cremasco V, Astarita JL. Immunological hallmarks of stromal cells in the tumour microenvironment. Nat Rev Immunol 2015;15:669-82.

7. Huo J, Hu J, Liu G, et al. Elevated serum interleukin-37 level is a predictive biomarker of poor prognosis in epithelial ovarian cancer patients. Arch Gynecol Obstet 2017;295:459-65.

8. Li L, Chen L, Zhang W, et al. Serum cytokine profile in patients with breast cancer. Cytokine 2017;89:173-8.

9. Lam SW, Nota NM, Jager A, et al. Angiogenesis- and Hypoxia-Associated Proteins as Early Indicators of the Outcome in Patients with Metastatic Breast Cancer Given First-Line Bevacizumab-Based Therapy. Clin Cancer Res 2016;22:1611-20.

10. Dirix LY, Vermeulen PB, Pawinski A, et al. Elevated levels of the angiogenic cytokines basic fibroblast growth factor and vascular endothelial growth factor in sera of cancer patients. Br J Cancer 1997;76:238-43.

11. Maemura M, Iino Y, Yokoe T, et al. Serum concentration of hepatocyte growth factor in patients with metastatic breast cancer. Cancer Lett 1998;126:215-20.

12. Gunel N, Coskun U, Sancak B, et al. Clinical importance of serum interleukin-18 and nitric oxide activities in breast carcinoma patients. Cancer 2002;95:663-7.

13. Dwyer RM, Potter-Beirne SM, Harrington KA, et al. Monocyte chemotactic protein-1 secreted by primary breast tumors stimulates migration of mesenchymal stem cells. Clin Cancer Res 2007;13:5020-7.

14. Lv M, Xiaoping X, Cai H, et al. Cytokines as prognstic tool in breast carcinoma. Front Biosci (Landmark Ed) 2011;16:2515-26.

15. Baskic D, Popovic S, Bankovic D, et al. Evaluation of inflammatory biomarkers as helping diagnostic tool in patients with breast cancer. Cancer Biomark 2014;14:401-8.

16. Noh SJ, Bae JS, Jamiyandorj U, et al. Expression of nerve growth factor and heme oxygenase-1 predict poor survival of breast carcinoma patients. BMC Cancer 2013;13:516.

Cite this article as: Huang H, Zhang Y, Li S, Zhao J, Wang X, Zhou X, Ren J. Multiplexed immunoassay-based serum cytokine profiling for potential prognosis predictors in patients with metastatic breast cancer. Transl Cancer Res 2018;7(6):15611566. doi: $10.21037 /$ tcr.2018.11.29 